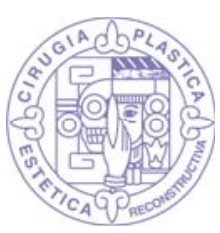

CASO CLÍNICO

doi: $10.35366 / 101084$

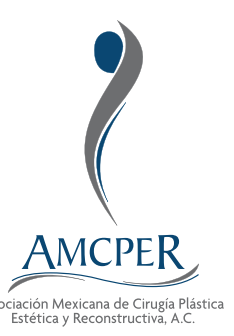

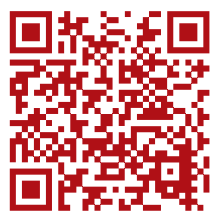

Palabras clave: Seroma, líquido periprotésico, implantes de pantorrilla, patologías relacionadas con implantes, rotura de implantes, complicaciones en implantes.

Keywords: Seroma, periprosthetic fluid, calf implants, implant-related

pathologies, implant rupture, implant complications.

* Cirujana Plástica, Hospital Ángeles Villahermosa. Miembro de la Asociación Mexicana de Cirugía Plástica, Estética y Reconstructiva. México. \$Cirujano Plástico, asesor del Comité de Seguridad de la Asociación Mexicana de Cirugía Plástica, Estética y Reconstructiva.

México.

$\S$ Radiólogo, Centro de Imagenología de Especialidades Médicas (CIEM). Villahermosa, Tabasco.

Recibido:

02 diciembre 2020

Aceptado:

20 enero 2021

\section{Seroma en pierna por implantes de pantorrilla}

\author{
Leg seroma due to calf implants
}

\author{
Dra. Lucrecia Arceo-Giorgana,* Dr. Jesús A Cuenca-Pardo, \\ Dr. Ángel Niño de Rivera-Hermosillo ${ }^{\S}$
}

\section{RESUMEN}

El seroma es una eventualidad que puede presentarse en pacientes con implantes mamarios. Es producido por diferentes etiologías y es una alerta de patologías mamarias relacionadas con los implantes mamarios. El estudio del líquido periprotésico es necesario para identificar la causa y dar un tratamiento adecuado. En la literatura médica no encontramos reportes de seroma en piernas por implantes de pantorrilla. Presentamos el caso de una paciente de 59 años de edad con implantes en piernas desde hace 28 años; presentó un seroma de cuatro años de evolución. Previo a la cirugía el volumen aumentó considerablemente, por lo que el implante fue retirado, el líquido evacuado y la cápsula resecada. Encontramos que el implante estaba roto y tenía una fuga de silicón intracapsular. Los especímenes fueron enviados a patología y al laboratorio para su estudio. El reporte mencionó una reacción xantogranulomatosa, con respuesta a cuerpo extraño. En los cultivos, incluyendo para micobacteria, no hubo desarrollo. Los hallazgos durante la cirugía y el reporte de patología ayudaron a deducir que la rotura del implante y la fuga del gel de silicón fueron la causa del seroma. La rotura y fuga de silicón es atribuible al tiempo prolongado de permanencia dentro de la paciente, a la actividad deportiva que realizaba y a los efectos de degradación.

\section{ABSTRACT}

Seroma is an eventuality that can occur in patients with breast implants. It can be produced by different etiologies and it is a warning of mammary pathologies related to mammary implants. The study of the periprosthetic liquid is necessary to identify the cause and give an adequate treatment. We were not able to find reports in the medical literature of seroma in the legs due to calf implants. We present a case of a 59-year-old patient with 28-year-old leg implants. She had seroma of four years of evolution. Prior to surgery the volume increased considerably, the implant was removed, the fluid evacuated and the capsule resected. We found the implant was broken and it had intracapsular silicone leak. The specimens were sent to pathology and to the laboratory for their study. The report mentioned a xanthogranulomatous reaction, with response to a foreign body. In cultures, including for mycobacteria, there was no development. The findings during surgery and the pathology report help us to deduce that the implant rupture and the silicone gel leak were the cause of the seroma. The breakage and leakage of silicone is attributable to the length of time in the patient, to the effects of degradation and the sports practiced by the patient.

\section{INTRODUCCIÓN}

$\mathrm{E}$ I seroma o líquido periprotésico puede ocurrir meses o años después de la colocación de un implante. ${ }^{1}$ Las causas del seroma son: idiopática, traumática, infecciosa, rotura del implante, metaplasia sinovial, desórdenes linfoproliferativos y linfoma anaplásico de células grandes asociado con implantes mamarios
(BIA-ALCL). ${ }^{1-15}$ Algunos autores han considerado que los esfuerzos pueden desprender el implante, causando microtraumatismos con pequeñas hemorragias y salida de líquido plasmático. Este mecanismo juega un papel importante en la fisiopatología del seroma mamario. 1,3,5,11-31

Los pacientes con seroma presentan asimetría mamaria por aumento de volumen en una de sus mamas; además, puede acompañarse de
Citar como: Arceo-Giorgana L, Cuenca-Pardo JA, Niño de Rivera-Hermosillo Á. Seroma en pierna por implantes de pantorrilla. Cir Plast. 2021; 31 (1): 31-38. https://dx.doi.org/10.35366/101084 
un proceso inflamatorio alrededor del implante. Con frecuencia se confunden las contracturas capsulares con roturas de los implantes. La rotura de implantes se puede acompañar de seroma; algunos datos clínicos son la pérdida de consistencia de una de las mamas y la presencia de adenomegalia axilar. La principal manifestación de los procesos linfoproliferativos como el linfoma de células gigantes asociados con implantes (BIA-ALCL) es el seroma. ${ }^{1,3-15}$ La infección del material implantado es otra de las causas frecuentes de seroma, pueden tener su origen en infecciones remotas como vías urinarias y garganta. Identificar la infección y dar tratamiento adecuado puede evitar esta complicación..$^{25-28}$

El seroma es una alerta de diversas patologías relacionadas con los implantes de silicón. El estudio cuidadoso del líquido periprotésico es básico en el diagnóstico y determinación de la etiología. ${ }^{32}$ El linfoma no Hodgkin tipo $B$ de células gigantes ha sido relacionado principalmente con los implantes mamarios; sin embargo, existen muchos reportes que lo han asociado con otros tipos, tales como prótesis de glúteos, inyecciones faciales de silicona, prótesis vasculares o prótesis bariátricas, prótesis óseas o vasculares. Existen algunas características similares en todos estos casos: la presencia de seroma, edema y dolor. Cuando el diagnóstico se hace temprano y el tratamiento oportuno hay una mejor evolución. El retiro de los implantes ofrece un mejor pronóstico a los pacientes afectados. ${ }^{33}$

Objetivo de la presentación: el seroma crónico es una manifestación que puede presentarse como una alerta de patologías relacionada con implantes mamarios o de rotura del dispositivo. En todos los casos es obligatorio realizar estudios de gabinete, laboratorio y patología para determinar la etiología; el seroma puede ser la primera manifestación de un linfoma. Los seromas y linfomas pueden presentarse con otro tipo de implantes. No encontramos referencias bibliográficas de incidencia en implantes cosméticos en pantorrillas, por lo que este caso podría ser el primero en ser reportado.

\section{CASO CLÍNICO}

Mujer de 59 años de edad, con antecedentes heredofamiliares de dos hermanos fallecidos por cáncer: uno sin especificar y el otro de cáncer hepático. Antecedentes personales no patológicos: de clase socioeconómica media; ocupación: gerente de una empresa hasta hace algunos años en que perdió su trabajo. La paciente realizó sus actividades cotidianas normales, incluyendo ejercicio durante 21 años, con nutrición alta en carbohidratos procesados y grasa saturada. Consumo tabáquico y alcohólico negativos. Antecedentes personales patológicos: sarampión y varicela en la infancia. Alérgicos y transfusionales negativos. Antecedentes ginecoobstétricos: dos gestas; dos para; fecha de la última menstruación hace 17 años. Antecedentes quirúrgicos: implantes de pantorrillas y de mentón en 1992.

\section{Medidas antropométricas:}

Estatura: $157 \mathrm{~cm}$; peso: $55.50 \mathrm{~kg}$; abdomen: $90 \mathrm{~cm}$; cadera: $92 \mathrm{~cm}$; porcentaje de grasa: 29.62 .

Signos vitales: T/A 110/70, FC $72 x^{\prime}, \mathrm{SatO}_{2} 97$.

En 1992 le colocaron implantes de pantorrilla marca Silimed lisos, de $24 \mathrm{~cm}$ de longitud por $5 \mathrm{~cm}$ de ancho, a nivel de hueco poplíteo en plano subfascial. Por 24 años permaneció asintomática. En el año 2016 refiere caer accidentalmente de una escalera al estar bajando frutos; no recuerda haberse golpeado las piernas. Días después notó aumento de volumen asintomático y progresivo de la pierna izquierda, permaneció en estas condiciones. En junio de 2020, sin causa aparente, se incrementó el volumen de manera exagerada, fue valorada el 14 de

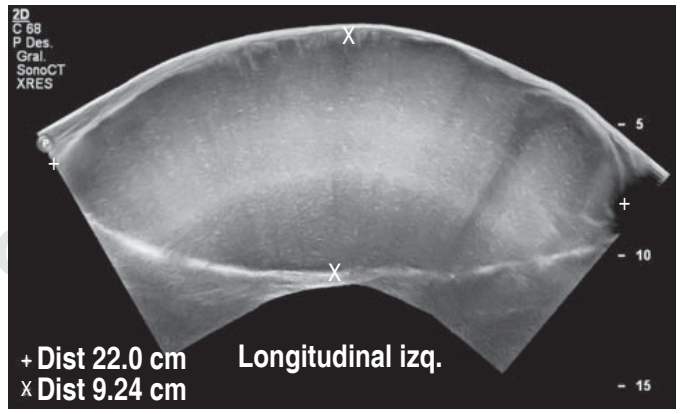

Figura 1: Imagen en eje largo del seroma del compartimiento posterior de la pantorrilla izquierda (señalado entre las $\mathrm{X} \mathrm{y}+$ ). 
octubre de 2020, encontrando buen estado general, tolerando la deambulación. La pierna derecha con el implante en su lugar, sin datos sugestivos de proceso inflamatorio. La pierna izquierda con aumento de volumen sin otros datos de inflamación. Se solicitaron exámenes de laboratorio y ultrasonido.

Resultado de los exámenes de laboratorio:

\section{Biometría hemática:}

Hemoglobina $(\mathrm{Hb}) 12.3$, hematocrito $(\mathrm{Hto})$ 41.2, plaquetas 173,000 , leucocitos 6,100, linfocitos $28 \%$, monocitos $8 \%$, segmentados $61 \%$, bandas $0 \%$.

Grupo sanguíneo $\mathrm{O} \mathrm{Rh}+$, tiempo de protrombina 13.09, porcentaje de actividad

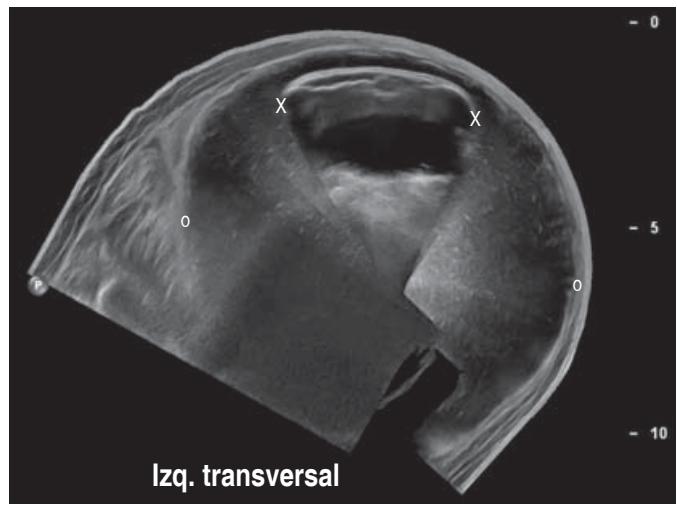

Figura 2: Imagen extendida en eje corto del compartimiento posterior de la pantorrilla izquierda en la que se observa el seroma $(\mathrm{O})$ y en el centro el implante $(\mathrm{X})$.

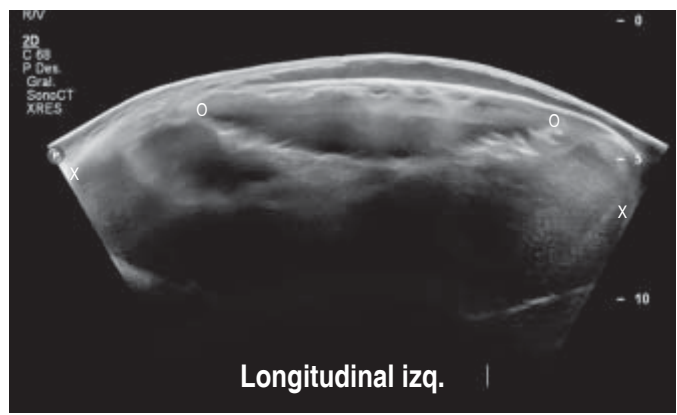

Figura 3: Imagen extendida en eje largo del compartimiento posterior de la pierna izquierda en la que se observa el seroma $(X)$ y en la parte superior el implante (O), su contorno anterior es irregular con pérdida de continuidad en relación con ruptura.

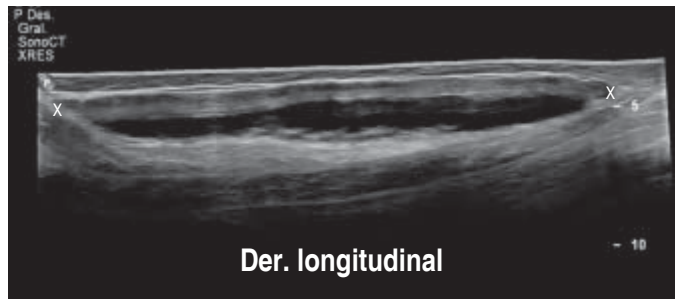

Figura 4: Imagen en eje largo del compartimiento posterior de la pantorrilla derecha. Se observa el implante (X) irregular, con datos de ruptura por tener su borde anterior de mayor ecogenicidad.

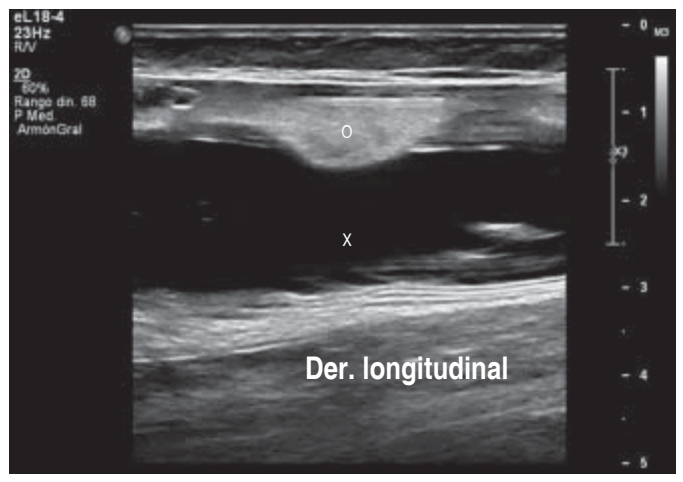

Figura 5: Detalle del compartimiento posterior de la pantorrilla derecha en el que se observa el implante (X) con una zona de pérdida de la continuidad de su pared $(\mathrm{O})$, con salida de material en relación con ruptura.

$84.5 \%$, índice internacional normalizado (INR) 1.07, tiempo de tromboplastina 1.07, $31.67 \mathrm{seg}$.

Anticuerpos anti-HIV 1 y 2: negativo.

\section{Química sanguínea:}

Glucosa $80 \mathrm{mg} / \mathrm{dL}$, urea 29.0, nitrógeno ureico 13.55, creatinina 0.87 , ácido úrico 2.2 $\mathrm{mg} / \mathrm{dL}$, colesterol $184 \mathrm{mg} / \mathrm{dL}$, triglicéridos 155 $\mathrm{mg} / \mathrm{dL}$, colesterol total $184 \mathrm{mg} / \mathrm{dL}$, lipoproteínas de alta densidad (HDL por sus siglas en inglés) $49 \mathrm{mg} / \mathrm{dL}$, lipoproteínas de baja densidad, (LDL por sus siglas en inglés) $98 \mathrm{mg} / \mathrm{dL}$.

Pruebas para SARS-CoV-2: PCR negativa, anti-SARS-CoV-2 (IgM) 0.5 negativo, anti-SARSCoV-2 (IgG) 0.35 negativo.

En el estudio de ultrasonido se encontró extensa colección líquida intracapsular de la pierna izquierda, sin descartar la ruptura del implante. El implante derecho con datos de ruptura y mínima colección. 


\section{Procedimiento quirúrgico (Figuras 1 a 5):}

El 24 de octubre se llevó a cabo el retiro del implante de la pantorrilla izquierda bajo anestesia local y sedación. El retiro del implante

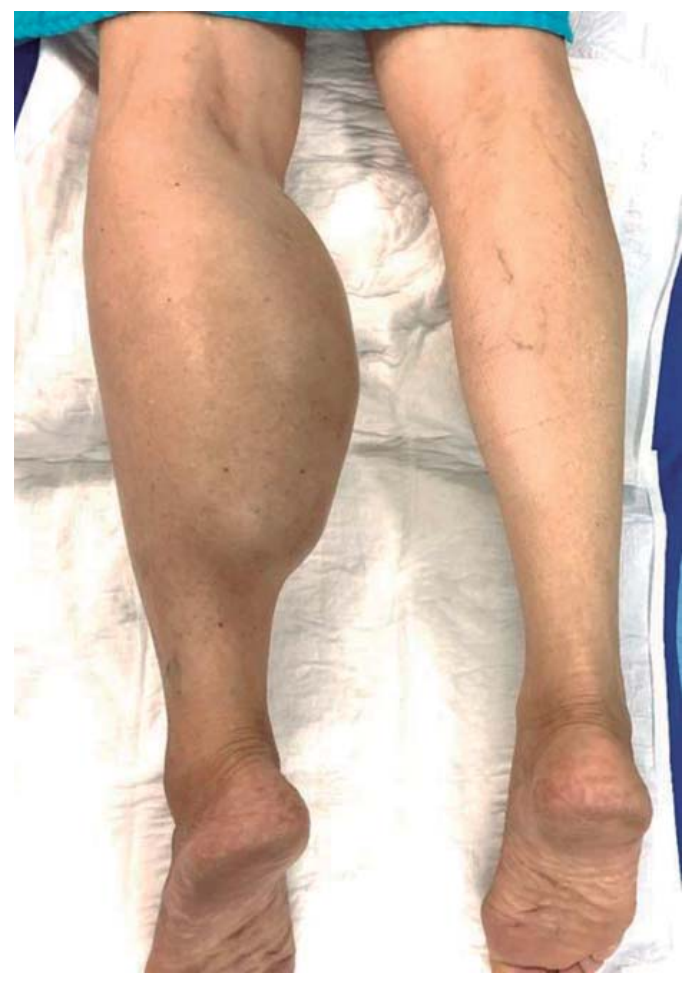

Figura 6: Aumento de volumen de la pierna izquierda. Imagen preoperatoria en decúbito ventral.

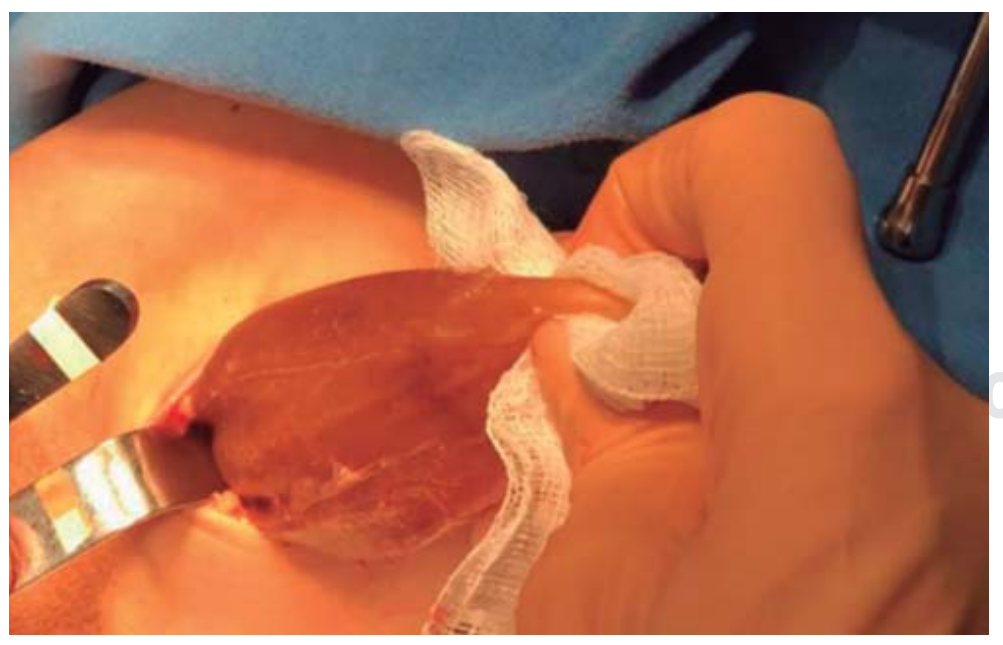

Figura 7: Extracción del implante. Se aprecia la fuga del gel de silicón.

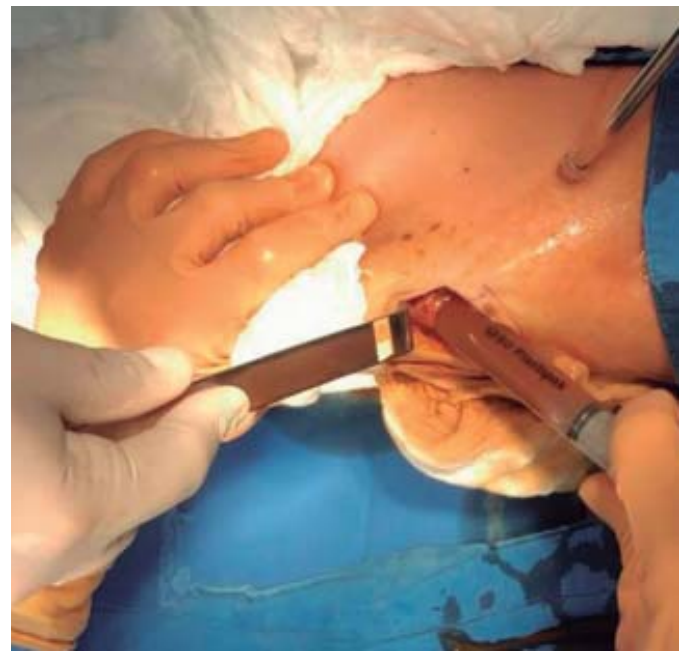

Figura 8: Líquido periprotésico: características físicas. Volumen aproximado total de $350 \mathrm{~mL}$.

derecho se difiere para otro tiempo quirúrgico (dos meses) a petición de la paciente para que se facilitara su movilidad en el postoperatorio. En la imagen preoperatoria observamos el aumento de volumen de la pierna izquierda (Figura 6).

Se realizó una incisión de $6 \mathrm{~cm}$ por debajo del hueco poplíteo, se disecaron los tejidos hasta identificar la cápsula del implante; al incidirla drenó aproximadamente $350 \mathrm{~mL}$ de líquido oscuro (achocolatado), inodoro. Se procedió a la extracción del implante, se encontró con varias perforaciones por donde se fugaba el gel de silicón y se resecó la cápsula (Figuras 7 a 9). En seguida se efectuó un aseo del bolsillo con solución fisiológica y agua oxigenada, se colocó un drenaje de silicón y se suturaron las heridas en dos planos: el tejido celular subcutáneo con vicryl 4-0 y la piel con nylon 4-0, para finalmente cubrirse con gasa y vendaje. El líquido y la cápsula fueron enviados a patología y laboratorio para su cultivo. Se manejó como paciente externa y se le prescribió ketorolaco, cefalosporina y etamsilato. Al cuarto día se le retiró el drenaje por haber drenado sólo $5 \mathrm{~mL}$ en 24 horas y color serohemático. Al décimo día se retiraron los puntos y se indicaron medias elásticas y deambulación.

A un mes de evolución las heridas están cerradas, no hay evidencia de recidiva del seroma; el edema de la pierna ha disminuido 
y la paciente está asintomática y deambulando (Figura 10).

En los resultados del hallazgo del cultivo no hay reporte de desarrollo bacteriano ni micótico. Asimismo, los hallazgos de patología de la cápsula muestran pared de tejido conectivo con fibrosis extensa, hemorragia reciente y antigua e inflamación crónica, xantogranulomatosa con células gigantes multinucleadas de tipo cuerpo extraño (a cristales de colesterol y material NO identificado), compatible con cápsula de seroma/derrame periimplante, negativo a neoplasia. La citología del líquido de derrame periimplante evidencia moderada cantidad de macrófagos reactivos y aislados linfocitos sobre un fondo proteináceo compatible con líquido de derrame, negativo a células neoplásicas. Resultados de inmunohistoquímica: CD30: negativo; CD68: positivo, macrófagos. Comentario del patólogo: los hallazgos morfológicos sugieren un proceso inflamatorio de larga evolución que pudo haber sido desencadenado por factores mecánicos o por filtración del contenido del implante. Se identificaron células atípicas aisladas, la tinción de inmunohistoquímica para CD30 fue negativa. Se descartó la posibilidad de un linfoma anaplásico de células grandes asociado con implantes.

\section{DISCUSIÓN}

Presentamos el caso de una paciente con implantes estéticos de silicón en piernas con

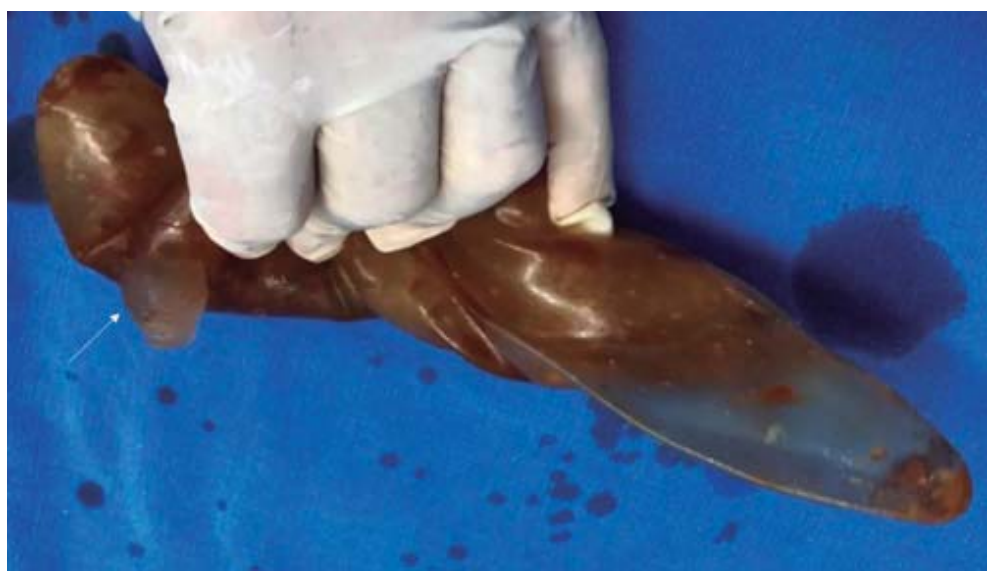

Figura 9: Rotura del implante con fuga de silicón y deflación.

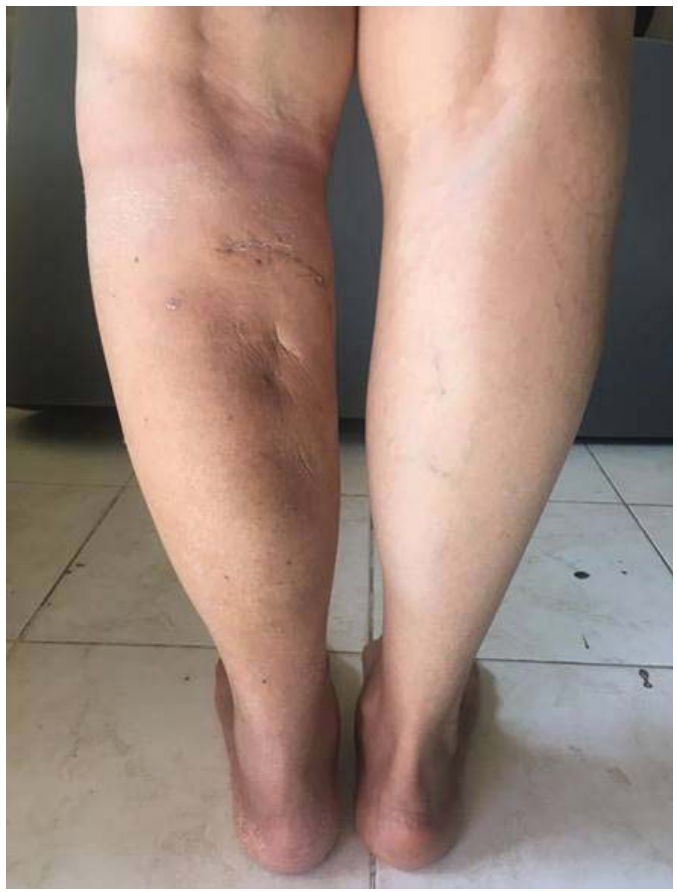

Figura 10: Aspecto postoperatorio a un mes y cuatro días.

una longevidad de 28 años. Después de un traumatismo formó un seroma en la pierna izquierda con cuatro años de evolución. El volumen aumentó considerablemente en los últimos meses. El seroma fue evacuado, la cápsula resecada y el implante retirado. Durante la cirugía se encontró una cápsula muy gruesa, líquido abundante y el implante roto, con fuga de silicón intracapsular. En los cultivos realizados del líquido periprotésico no hubo desarrollo de gérmenes. Los estudios de patología demostraron un proceso inflamatorio xantogranulomatoso con respuesta a cuerpo extraño. Aunque el estudio de patología no es el medio para identificar el tipo de cuerpo extraño, la respuesta encontrada debe ser atribuida a las micropartículas de silicón por la fuga del gel por la rotura del implante.

Los seromas, los procesos linfoproliferativos y los linfomas han sido relacionados principalmente con implantes mamarios, algunos pocos casos a otro tipo de implantes, incluyendo de glúteos. ${ }^{2,11-23,32,33}$ Sin embargo, no hay casos reportados en pantorrilla. Nosotros reportamos un caso de pantorrilla. El estudio y tratamiento debe ser similar en todos los casos. 
Los implantes en el organismo sufren de una degradación por efectos de los glóbulos blancos, enzimas y radicales libres de oxígeno que desgastan, rompen y facilitan la fuga de micropartículas de silicón; los polímeros se transforman en polímeros simples y sílice. Se ha reportado el desprendimiento de micropartículas de los implantes mamarios y no mamarios, éstas han sido involucradas en la respuesta linfoproliferativa y en el desarrollo de diferentes patologías, incluyendo los linfomas. El tiempo de permanencia de los implantes es uno de los factores más importantes en el desgaste de los mismos. Los esfuerzos físicos pueden desprender el implante causando microtraumatismos y formación de seroma. ${ }^{1,3,5,11-31,34-39}$ El caso que presentamos tenía 28 años con sus implantes. El desgaste por la acción enzimática de los leucocitos debió ser muy importante. La paciente realizaba en forma rutinaria ejercicios y cuatro años previos a la cirugía sufrió un traumatismo. Estos factores debieron contribuir a la rotura de los implantes.

El seroma es una alerta de patologías relacionadas con implantes, puede ser ocasionado por diferentes causas, incluyendo la rotura. El estudio del líquido deberá ser efectuado para identificar su etiología. ${ }^{40-55}$ En los estudios realizados a los especímenes obtenidos durante la cirugía no se encontró evidencia de proceso infeccioso ni de malignidad. La respuesta xantogranulomatosa con reacción a cuerpo extraño fue provocada por la rotura de los implantes y la fuga del gel de silicón. El proceso inflamatorio que estaba ocasionando el seroma fue controlado con el retiro del implante, la resección de la cápsula y la extracción del líquido.

\section{CONCLUSIÓN}

El seroma es una manifestación de alerta de diferentes patologías relacionadas con los implantes mamarios y no mamarios. Todas las pacientes con implantes deberán tener una vigilancia estrecha por los cirujanos, una revisión periódica con exploración dirigida para buscar alteraciones relacionadas con sus implantes y estudios de ultrasonido de alta resolución y/o resonancia magnética. Estas recomendaciones harán una mayor detección de los seromas y ayudarán a comprender su impacto en la salud de las pacientes. El estudio del líquido acumulado alrededor de los implantes será de mucha utilidad para determinar la etiología de los seromas.

El caso que presentamos se trata de una paciente con un seroma en la pierna izquierda, aunque el reporte de patología no puede determinar la etiología exacta, los hallazgos durante la cirugía y el reporte de patología de una reacción xantogranulomatosa con respuesta a cuerpo extraño nos orienta a deducir que la rotura del implante y la fuga del gel de silicón fueron la causa.

La rotura y fuga de silicón es atribuible al largo tiempo de permanencia dentro de la paciente, a la actividad deportiva que realizaba y a los efectos de degradación.

\section{REFERENCIAS}

1. Lista F, Ahmad J. Evidence-based medicine: augmentation mammaplasty. Plast Reconstr Surg 2013; 132 (6): 1684-1696.

2. Ronchi A, Montella M, Argenzio V, Lucia A, De Renzo A, Alfano R et al. Diagnosis of anaplastic large cell lymphoma on late peri-implant breast seroma: management of cytological sample by an integrated approach. Cytopathology 2018; 29 (3): 294-299.

3. Derby BM, Codner MA. Textured silicone breast implant use in primary augmentation: core data update and review. Plast Reconstr Surg 2015; 135: 113-124.

4. Lista F, Tutino R, Khan A, Ahmad J. Subglandular breast augmentation with textured, anatomic, cohesive silicone implants: a review of 440 consecutive patients. Plast Reconstr Surg 2013; 132 (2): 295-302.

5. Bengtson B, Brody GS, Brown MH, Glicksman C, Hammond D, Kaplan $\mathrm{H}$ et al. Managing late periprosthetic fluid collections (seroma) in patients with breast implants: a consensus panel recommendation and review of the literature. Plast Reconstr Surg 2011; 128 (1): 1-7.

6. Jordan SW, Khavanin N, Kim JYS. Seroma in prosthetic breast reconstruction. Plast Reconstr Surg 2016; 137 (4): 1104-1116.

7. Brohim RM, Foresman PA, Hildebrandt PK, Rodeheaver GT. Early tissue reaction to textured breast implants surfaces. Ann Plast Surg 1992; 28: 354-362.

8. Malata CM, Feldberg L, Coleman DJ, Foo IT, Sharpe DT. Textured or smooth implants for breast augmentation? Three year follow-up of a prospective randomized controlled trial. Br J Plast Surg 1997; 50: 99-105.

9. Coleman DJ, Foo IT, Sharpe DT. Textured or smooth implants for breast augmentation? A prospective controlled trial. Br J Plast Surg 1991; 44: 444-448.

10. Collis N, Coleman D, Foo IT, Sharpe DT. Ten-year review of a prospective randomized controlled trial of textured versus smooth subglandular silicone gel breast implants. Plast Reconstr Surg 2000; 106: 786-791. 
11. Hall-Findlay EJ. Breast implant complication review: double capsules and late seromas. Plast Reconstr Surg 2011; 127: 56-66.

12. Spear SL, Rottman SJ, Glicksman C, Brown M, Al-Attar A. Late seromas after breast implants: Theory and practice. Plast Reconstr Surg 2012; 130: 423-435.

13. Bengtson B, Brody GS, Brown MH, Glicksman C, Hammond D, Kaplan $\mathrm{H}$ et al. Late periprosthetic fluid collection after breast implant working group. Managing late periprosthetic fluid collections (seroma) in patients with breast implants: A consensus panel recommendation and review of the literature. Plast Reconstr Surg 2011; 128: 1-7.

14. Santanelli di Pompeo F, Laporta R, Sorotos M, Di Napoli A, Giovagnoli MR, Cox MC et al. Breast implant-associated anaplastic large cell lymphoma: Proposal for a monitoring protocol. Plast Reconstr Surg 2015; 136: 144e-151e.

15. Di Napoli A. Achieving reliable diagnosis in late breast implant seromas: from reactive to anaplastic large cell lymphoma. Plast Reconstr Surg 2019; 143: 15S-22S.

16. Becker H, Klimczak J. Aspiration of periprosthetic seromas using the blunt seroma cath. Plast Reconstr Surg 2016; 137 (2): 473-475.

17. Chourmouzi D, Vryzas T, Drevelegas A. New spontaneous breast seroma 5 years after augmentation: a case report. Cases J 2009; 2: 7126.

18. Fodor L, Moscona R. Late post-traumatic intracapsular seroma after breast augmentation. J Plast Reconstr Aesthet Surg 2009; 62: e609-e610.

19. Hasham S, Akhtar S, Fourie LR. Persistent seroma following breast prosthesis explantation: A case report and review. Eur J Plast Surg 2006; 28: 490-493.

20. Mazzocchi M, Dessy LA, Carlesimo B, Marchetti F, Scuderi N. Late seroma formation after breast surgery with textured silicone implants: a problem worth bearing in mind. Plast Reconstr Surg 2010; 125: 176e-177e.

21. Mazzochi M, Dessy LA, Corrias F, Scuderi N. A clinical study of late seroma in breast implantation surgery. Aesthet Plast Surg 2012; 36 (1): 97-104.

22. Oliveira VM, Roveda Junior D, Lucas FB, Lucarelli AP, Martins MM, Rinaldi JF et al. Late seroma after breast augmentation with silicone prostheses: a case report. Breast J 2007; 13: 421-423.

23. Tansley PD, Powell BW. Late swelling after bilateral breast augmentation. J Plast Reconstr Aesthet Surg 2011; 64: 261-263.

24. Pinchuk V, Tymofii O. Seroma as a late complication after breast augmentation. Aesthet Plast Surg 2011; 35 (3): 315-318.

25. Bumaschny E, Rafa CA, Reichman P. Evaluación preoperatoria del paciente quirúrgico. En: Galindo $\mathrm{F}$ y cols. Enciclopedia de cirugía digestiva. tomo I-101, 2013. pp. 1-20.

26. Guía de referencia rápida. Diagnóstico y tratamiento de la infección aguda, no complicada del tracto urinario en la mujer. Guía de Práctica Clínica IMSS-077-08.

27. López Tagle D, Hernández Ferrer M, Saldivar Arias T, Sotolongo Hernández T, Valdés Dupeyrón O. Infección de la herida quirúrgica: Aspectos epidemiológicos. Rev Cub Med Mil [Internet] 2007; 36 (2). Disponible en: http://scielo.sld.cu/scielo.php?script =sci arttext\&pid=S0138-65572007000200008\&lng=es.
28. Pineda V, Caceres J, Pernas JC, Catala J. Retromammary fluid collection as a late complication of breast implants: magnetic resonance imaging findings. J Comput Assist Tomogr 2004; 28: 386-389.

29. Bengtson BP, Eaves FF III. High-resolution ultrasound in the detection of silicone gel breast implant shell failure: background, in vitro studies, and early clinical results. Aesthet Surg J 2012; 32: 157-174.

30. Chung KC, Malay S, Shauver MJ, Kim HM. Economic analysis of screening strategies for rupture of silicone gel breast implants. Plast Reconstr Surg 2012; 130: 225-237.

31. McCarthy CM, Pusic AL, Kerrigan CL. Silicone breast implants and magnetic resonance imaging screening for rupture: Do U.S. Food and Drug Administration recommendations reflect an evidence-based practice approach to patient care? Plast Reconstr Surg 2008; 121 (4): 1127-1134.

32. Cuenca-Pardo J, Vélez-Benítez E, Ramos-Gallardo G, Iribarren-Moreno R. Seroma una alerta de patologías relacionadas a implantes mamarios MBE. Cir Plast 2019; 29 (1): 12-22.

33. Ramos-Gallardo G, Carballo-Zarate AA, Cuenca-Pardo J, Cárdenas-Camarena L, Solano Genesta M, Beltrán JAC et al. What is the evidence of lymphoma in patients with prostheses other than breast implants? Aesthet Plast Surg 2020; 44 (2): 286-294.

34. Palraj B, Paturi A, Stone RG, Alvarez H, Sebenik M, Perez MT et al. Soft tissue anaplastic large T-cell lymphoma associated with a metallic orthopedic implant: case report and review of the current literature. J Foot Ankle Surg 2010; 49: 561-564.

35. Menter T, Ballova V, Caspar C, Wolff T, Kasend B, Singer $\mathrm{G}$ et al. ALK-negative anaplastic large cell lymphoma arising in the thrombus of an aortic prosthesis preceded by clonally related lymphomatoid papulosis. Virchows Archiv 2019; 474: 763-767.

36. Suhail-Chaudhry M, Mather H, Marks A, Naresh K. Diffuse large B cell lymphoma complicating total knee arthroplasty: case report and literature review of the association of diffuse large B cell lymphoma with joint replacement. Acta Haematol 2011; 126: 141-146.

37. Rajeev A, Ralte A, Choudhry N, Jabbar F, Banaszkiewicz P. Diffuse B cell non-Hodgkin's lymphoma presenting atypically as periprosthetic joint infection in a total hip replacement. Case Rep Orthop 2017; 2017: 7195016.

38. McCarthy CL, Uchihara Y, Vlychou M, Grammatopoulos G, Athanasou NA. Development of malignant lymphoma after metal-on-metal hip replacement: a case report and review of the literature. Skeletal Radiol 2017; 46 (6): 831-836.

39. Hallab NJ, Samelko L, Hammond D. The inflammatory effects of breast implant particulate shedding: comparison with orthopedic implants. Aesthet Surg J 2019; 39 (S1): 36-S48.

40. Song JW, Kim HM, Bellfi LT, Chung KC. The effect of study design biases on the diagnostic accuracy of magnetic resonance imaging for detecting silicone breast implant ruptures: a meta-analysis. Plast Reconstr Surg 2011; 127: 1029-1044.

41. Handel N, Garcia ME, Wixtrom R. Breast implant rupture: causes, incidence, clinical impact and management. Plast Reconstr Surg 2013; 132: 1128-1137. 
42. Gunawardana RT, Dessauvagie BF, Taylor DB. Breast implant-associated anaplastic large cell lymphoma, an under-recognized entity. J Med Imaging Radiat Oncol 2019; 63: 630-638.

43. Leberfinger AN, Behar BJ, Williams NC, Rakszawski KL, Potochny JD, Mackay DR et al. Breast implantassociated anaplastic large cell lymphoma: a systematic review. JAMA Surg 2017; 152 (12): 1161-1168.

44. Fitzal F, Turner SD, Kenner L. Is breast implantassociated anaplastic large cell lymphoma a hazard of breast implant surgery? Open Biol 2019; 9 (4): 190006. doi: 10.1098/rsob.190006.

45. Clemens MW, Jacobsen ED, Horwitz SM. 2019 NCCN consensus guidelines on the diagnosis and treatment of breast implant-associated anaplastic large cell lymphoma (BIA-ALCL). Aesthet Surg J 2019; 39 (S1): S3-S13.

46. Mazzocchi M, Dessy LA, Marchetti F, Marchetti F Carlesimo B. The use of the Veress needle to drain mammary periprosthetic fluid. In Vivo 2010; 24 : 219-222.

47. Garcia-Tutor E, Murillo J. Safe drainage by puncture of post mastectomy seroma in a patient with immediate reconstruction using an expander. Plast Reconstr Surg 2003; 111: 1357-1358.

48. Moyer KE, Potochny JD. Technique for seroma drainage in implant-based breast reconstruction. J Plast Reconstr Aesthet Surg 2012; 65: 1614-1617.

49. Ramos-Gallardo G, Cuenca-Pardo J, RodríguezOlivares E, Iribarren-Moreno R, Contreras-Bulnes L, Vallarta-Rodríguez A et al. Breast implant and anaplastic large cell lymphoma meta-analysis. J Invest Surg 2017; 30: 56-65.

50. Pinsolle V, Grinfeder C, Mathoulin-Pelissier S, Faucher A. Complications analysis of 266 immediate breast reconstructions. J Plast Reconstr Aesthet Surg 2006; 59: 1017-1024.

51. Francis SH, Ruberg RL, Stevenson KB, Beck CE, Ruppert AS, Harper JT et al. Independent risk factors for infection in tissue expander breast reconstruction. Plast Reconstr Surg 2009; 124: 1790-1796.

52. Nahabedian MY, Tsangaris T, Momen B, Manson P. Infectious complications following breast reconstruction with expanders and implants. Plast Reconstr Surg 2003; 112: 467-476.

53. Rubino C, Brongo S, Pagliara D, Cuomo R, Abbinante $G$ et al. Infections in breast implant: a review a focus and developing countries. J Infect Dev Ctries 2014; 8 (9): 1089-1095.

54. Washer LL, Gutowski K. Breast implant infections. Infect Dis Clin N Am 2012; 26: 111-125.

55. Cuenca-Pardo J, Ramos-Gallardo G, ContrerasBulnes L, Iribarren-Moreno R, Rodríguez-Olivares E, Hernández-Valverde $\mathrm{C}$ et al. Factores relacionados con infecciones en implantes mamarios. Cir Plast 2015; 25 (1): 6-14.

Conflicto de intereses: Los autores declaran no tener conflicto de intereses.

Correspondencia:

Dra. Lucrecia Arceo-Giorgana

Hospital Ángeles Villahermosa.

Av. Prol. Paseo Usumacinta Núm. 2085,

consultorio 347-348, Col. Galaxia

Tabasco 2000, Villahermosa, Tabasco.

E-mail: I_arceo@hotmail.com 